\title{
Numerical models of broad-bandwidth nanosecond optical parametric oscillators
}

\author{
A. V. Smith and Russell J. Gehr \\ Dept. 1128 Lasers, Optics and Remote Sensing, Sandia National Laboratories, Albuquerque, NM \\ $87185-1423$
}

Mark S. Bowers

Aculight Corp., 11805 North Creek Parkway S., Suite 113 Bothell, WA 98011

Abstract

We present three new methods for modeling broad-bandwidth, nanosecond optical parametric oscillators in the plane-wave approximation. Each accounts for the group-velocity differences that determine the operating linewidth of unseeded optical parametric oscillators, and each allows the signal and idler waves to develop from quantum noise. The first two methods are based on split-step integration methods in which nonlinear mixing and propagation are calculated separately on alternate steps. One method relies on Fourier transforming the fields between $t$ and $\omega$ to handle propagation, with mixing integrated over a $\Delta z$ step: the other transforms between $z$ and $k_{z}$ in the propagation step, with mixing integrated over $\Delta t$. The third method is based on expansion of the three optical fields in terms of their respective longitudinal empty cavity modes, taking into account the carity boundary conditions. Equations describing the time development of the mode amplitudes are solved to yield the time dependence of the three output fields. These plane-wave models exclude diffractive effects, but can be readily extended to include them.

Sandia is a multiprogram laboratory operated by Sandia Corporation, a Lockheed Martin Company, for the United States Department of Energy under contract DE-AC04-94AL85000. 


\section{INTRODUCTION}

In recent years the discovery of new nonlinear optical materials, improvements in the performance of solid-state pump lasers, and progress in high-damage-threshold optics has stimulated renewed interest in optical parametric oscillators (OPO's). Despite recent progress, these devices have yet to reach their full potential as sources with broad tunability, narrow linewidth, and good beam quality. One reason is their behavior is more complex than is often appreciated. This complexity makes computer simulations essential in interpreting laboratory observations and in optimizing OPO designs. We reported earlier on a numerical model ${ }^{1}$ of nanosecond, pulsed OPO's that included diffraction. birefringent walk off, and pump de-

pletion. Its predictions agreed well with detailed measurements of spatial beam profiles and beam quality, spatially-resolved temporal pulse shapes, and frequency spectra for a singlefrequency, injection-seeded KTP ring OPO. The primary limitation of that model was its assumption of narrow bandwidth operation which is appropriate for injection-seeded, singlelongitudinal-mode devices pumped by single-longitudinal-mode pulses, but not for broad bandwidth operation or for multi-longitudinal-mode pumps. To address this shortcoming, we present here three new methods for modeling multimode, or broad-bandwidth OPO's . pumped by single-mode or multimode lasers, in the plane-wave limit. Each method can be extended to include diffraction and birefringent walk off, but that work is deferred, in part due to the computing power required for such a complete model.

These models of broad-bandwidth OPO's should prove useful in understanding the spectral properties of unseeded, or free running, pulsed OPO's that start from quantum noise, and also in understanding the conditions necessary for injection seeding them. For example, we noted in an earlier paper ${ }^{2}$ that a free-running: pulsed OPO generated broadband light with nearly pure phase modulation. The models presented here can test the generality of this observation. They can also be useful in predicting line widths and efficiencies of free running OPO's, and with realistic quantum noise models, they can predict the pulse-to-pulse fluctuations in output spectra and conversion efficiency. They should also answer questions (n) 


\section{DISCLAIMER}

This report was prepared as an account of work sponsored by an agency of the United States Government. Neither the United States Government nor any agency thereof, nor any of their employees, make any warranty, express or implied, or assumes any legal liability or responsibility for the accuracy, completeness, or usefulness of any information, apparatus, product, or process disclosed, or represents that its use would not infringe privately owned rights. Reference herein to any specific commercial product, process, or service by trade name, trademark, manufacturer, or otherwise does not necessarily constitute or imply its endorsement, recommendation, or favoring by the United States Government or any agency thereof. The views and opinions of authors expressed herein do not necessarily state or reflect those of the United States Government or any agency thereof. 


\section{DISCLAIMER}

Portions of this document may be illegible in electronic image products. Images are produced from the best available original document. 
of interest in injection seeding: such as the tolerance for detuning of the seed light from a carity mode; tolerance to phase mismatch in the crystal; comparison of seeding with $\mathrm{cw}$ and pulsed light; the influence of the pump pulse's temporal shape and energy: and the role of pump bandwidth in seeding. A general model to address these issues should permit multimode pump light, arbitrary mirror reflectivities, a realistic quantum noise model, arbitrary seed light properties, frequency selective cavity elements, and variable crystal properties. As we will show; our models meet these criteria.

We have chosen the OPO diagrammed in Fig. 1 as the target for our models, although the extension to other configurations is straightforward. The model must integrate the signal, idler. and pump wave equations through the crystal including nonlinear source terms, and it must apply appropriate boundary conditions at the mirrors and crystal interfaces.

\section{i.}

We define the signal field in terms of a carrier frequency $\omega_{s}$ by

$\left.E_{s} i t . z\right)=\frac{1}{2}\left\{\varepsilon_{s}(t, z) \exp \left[-i\left(\omega_{s} t-k_{s} z\right)\right]+\hat{\epsilon}_{s}^{*}(t ; z) \exp \left[i\left(\omega_{s} t-k_{s} z\right)\right]\right\}$.

so remporal and spatial structure is described by the coefficient $\varepsilon_{s}(t, z)$. Outside the crystal $k_{s}=\omega_{s} / c$, and inside the crystal $k_{s}=n_{s} \omega_{s} / c$, where $n_{s}$ is the crystal index of refraction at the signal wavelength. Similar relations describe the idler and pump waves. The boundary conditions simply require that at the input mirror the field amplitudes of the output waves are the sum of reflected input and transmitted circulating field amplitudes.

$\varepsilon_{j}^{o u s}(t, z=0)=\sqrt{1-R_{j}} \varepsilon_{j}^{c i r \hat{c}}(t, z=L) \exp \left[i \phi_{j}\right]-\sqrt{R_{j}} \varepsilon_{j}^{i n}(t . z=0)$.

anci the circulating amplitudes are the combination of the transmitted input amplitudes and the reflected circulating amplitudes

$\varepsilon_{j}^{\text {cirs }}(t, z=0)=\sqrt{1-R_{j}} \varepsilon_{j}^{i n}\left(t_{:} z=0\right)+\sqrt{R_{j}} \varepsilon_{j}^{\text {circ }}(t . z=L) \exp \left[i O_{j}\right]$.

where $j=s, i, p$ for signal, idler, or pump, and the $R_{j}$ 's are the reflectivities of the input mirror. The $z$ values 0 and $L$ refer to positions just after and just before the input mirror, and the phases $\phi_{j}$ are residual round-trip phases proportional to the detuning of the carrier 
waves from cavity resonance. At the crystal input face the field in the crystal is related to the circulating field by

$\varepsilon_{j}\left(t, z=z_{i n}\right)=\sqrt{\frac{1-R_{j}^{i n}}{n_{j}}} \varepsilon_{j}^{c i r c}\left(t, z=z_{i n}\right)$.

and at the exit face the relation is

$\varepsilon_{j}^{\text {circ }}\left(t, z=z_{\text {out }}\right)=\sqrt{\left(1-R_{j}^{\text {out }}\right) n_{j}} \varepsilon_{j}\left(t, z=z_{\text {out }}\right)$.

The three-wave parametric mixing equations that must be integrated inside the crystal are well known ${ }^{3}$ to reduce in the plane-wave limit to

$$
\begin{aligned}
& {\left[\frac{\partial}{\partial z}+\frac{1}{v_{s}} \frac{\partial}{\partial t}+i \alpha_{s} \frac{\partial^{2}}{\partial t^{2}}\right] \varepsilon_{s}(z, t)=\frac{i \omega_{s} d_{\mathrm{eff}}}{n_{s} c} \varepsilon_{p}(z, t) \varepsilon_{i}^{*}(z \cdot t) \exp (i \Delta k z) \equiv P_{s}(z, t)} \\
& {\left[\frac{\partial}{\partial z}+\frac{1}{v_{i}} \frac{\partial}{\partial t}+i \alpha_{i} \frac{\partial^{2}}{\partial t^{2}}\right] \varepsilon_{i}(z, t)=\frac{i \omega_{i} d_{\mathrm{eff}}}{n_{i} c} \varepsilon_{p}(z, t) \varepsilon_{s}^{*}(z, t) \exp (i \Delta k z) \equiv P_{i}(z, t)} \\
& {\left[\frac{\partial}{\partial z}+\frac{1}{v_{p}} \frac{\partial}{\partial t}+i \alpha_{p} \frac{\partial^{2}}{\partial t^{2}}\right] \varepsilon_{p}(z, t)=\frac{i \omega_{p} d_{\mathrm{eff}}}{n_{p} c} \varepsilon_{i}(z, t) \varepsilon_{s}(z, t) \exp (-i \Delta k z) \equiv P_{p}(z, t)}
\end{aligned}
$$

where $\Delta k$ is the phase velocity mismatch for the carrier waves defined by

$\Delta k=\dot{k_{p}}-k_{s}-k_{i}$

and the carrier frequencies satisfy

$\omega_{p}=\omega_{s}+\omega_{i}$

The second terms on the left in Eqs. (6-8) are associated with the group velocity, $v$, of each wave, defined by

$$
\frac{1}{v}=\frac{n}{c}+\frac{\omega}{c} \frac{d n}{d \omega}=\frac{d k}{d \omega}
$$

The third term describes group-velocity dispersion and is usually important only for bandwidths greater than several hundred wavenumbers. Because this is seldom the case for 
nanosecond OPO's except near degeneracy for type 1 mixing, we disregard that term throughout this paper.

In our previous model of single-longitudinal-mode OPO's; we ignored group velocity differences among the three waves, in effect assuming that all three waves propagate with equal group velocity. This was appropriate for near-monochromatic operation where the time structure on the optical fields was limited to a scale much longer than any of the group velocity walk off times defined by

$\tau_{j k}=L_{c}\left(\frac{1}{v_{j}}-\frac{1}{v_{k}}\right)$

where $L_{c}$ is the crystal length. For a broad-bandwidth OPO, in contrast, the waves are structured on a time scale as short as the signal-idler walk off time, $\tau_{s i}$, so the group velocity terms must be retained. In fact, it is the time slippage between the signal and idler waves due to their differing group velocities that is responsible for smoothing structure over times shorter than $\tau_{s i}$, limiting the operating bandwidth of a free running OPO to about $\tau_{s i}^{-1}$ or less. Our new models integrate Eqs. (6-8), retaining the group velocity term.

There are a few published theoretical treatments of multimode OPO's ${ }^{4-8}$. However, most neglect the group velocity term and consequently they provide a poor physical description of broad-bandwidth OPO's, although in some cases they predict spectral band widths reasonably well. Several of the models ${ }^{4-7}$ are based on an early theory of OPO's by Yariv and Louisell ${ }^{9}$. This analysis starts with three coupled first-order equations of motion for longitudinally space-averaged field amplitudes derived by expanding the intracavity signal. idler, and pump fields in a set of lossless and orthonormal cavity modes. The resulting rate equations are supposed to describe the time development of these fields in the case of single-mode operation with a single-mode pump. Cassedy and Jain ${ }^{4}$, Kong et al. ${ }^{5}$, Schroder et $a l^{6}$, and Boller and Schroder ${ }^{7}$ adapted these rate equations to the case of multiple signal and idler modes, assuming a single pump mode. However, it has been shown by numerous experiments and models ${ }^{1,10,11}$ that the assumption of spatially uniform fields is violated for most nanosecond OPO's. Generally, the pump beam is not resonated and is severely de- 
pleted as it passes through the crystal. Additionally, the idler wave is often unresonated and must grow from zero at the input end of the crystal to its output value. Even the resonated signal wave is nonuniform because the output coupling in nanosecond OPO's is high, typically 0.1 to 0.5 . To allow for nonuniform fields, Fix and Wallenstein ${ }^{8}$ modified this approach by using the mixing equations above without the group velocity terms. A single-mode pump was assumed but $N$ pairs of signal and idler modes were allowed with a value of $\Delta k$ assigned to each pair. The $(2 N+1)$ equations corresponding to Eqs. (6-8) above were integrated in $z$, assuming equal group velocities for all waves, and applying the boundary conditions at the mirrors on each round trip of the cavity. One problem with this method is that it does not properly handle group velocity effects. The $\Delta k$ 's appear only in the nonlinear polarization terms and describe the phase adjustments due to nonlinear mixing but not those due to linear propagation. All temporal structure that develops on the pulses is assumed to propagate with the same velocity for the three waves rather than with their actual group velocities. This method has been successful in predicting the linewidths of unseeded nanosecond OPO's, but a more realistic model that includes group velocity differences is needed to accurately describe the full range of broadband OPO behavior, including the effects of injection seeding. Only the model recently presented by Arisholm ${ }^{12}$ includes the group velocities. He treats them in an approximate way and he also incorporates start up from quantum noise fields.

In contrast to Fix and Wallenstein ${ }^{8}$, we follow the time development of only three waves, the carrier waves for the signal, idler, and pump. Spectra are deduced from the timedependent structure that develops on these waves. Generally, the structure will be nearly periodic with a cavity round-trip repetition time. corresponding to the usual longitudinal cavity modes. The time structure will be smoothed over a time scale of $t_{s i}$, the signal/idler walk off time, limiting the spectral width to the crystal's acceptance bandwidth or less. Using this approach. Equations (6-8), including the group velocity term, have successfully described single-pass parametric mixing of broad-bandwidth pulses ${ }^{13-18,20,22,23}$ as well as synchronously pumped OPO' ${ }^{24}$. Two common methods of numerical solution are the Runge-Kutta direct 
integration method as outlined by Bakker et al. ${ }^{13}$, and the split-step integration method in which linear propagation steps are alternated with nonlinear mixing steps. We present two examples of the latter method. We also present a method of solution based on the exact cavity equations of motion, which are derived by expanding the fields in a Fourier series of longitudinal modes. Our mode-expansion formulation allows for spatially non-uniform fields, arbitrary mirror reflectivities, and group velocity mismatch. All three models described below give identical results for simulating broad-band OPO's in the plane-wave limit.

\section{SPLIT-STEP METHOD 1}

In our first implementation of the split-step method, the field $\left(\varepsilon_{j}(t, z+\Delta z)\right.$ is determined from $\varepsilon_{j}(t, z)$ by first integrdting the equations without the nonlinear source term. In a reference frame with time transformed to keep the pulse with the fastest group velocity, $v_{m}$, stationary in time, defined by

$z^{\prime}=z$

$t^{\prime}=t-z / v_{m}$

the propagation equations inside the crystal have the form

$\left[\frac{\partial}{\partial z}+\left(\frac{1}{v_{j}}-\frac{1}{v_{m}}\right) \frac{\partial}{\partial t^{\prime}}\right] \varepsilon_{j}\left(z, t_{-}^{\prime}\right)=0$

Fourier transforming from $t^{\prime}$ to $\omega$ converts this equation to

$\left[\frac{\partial}{\partial z}+i \Delta \omega_{j}\left(\frac{1}{v_{j}}-\frac{1}{v_{m}}\right)\right] \varepsilon_{j}\left(z, \Delta \omega_{j}\right)=0$

where

$\varepsilon_{j}\left(\tilde{z}, \Delta \omega_{j}\right)=\int_{-\infty}^{\infty} \varepsilon_{j}\left(z, t^{\prime}\right) \exp \left[i \Delta \omega_{j} t^{\prime}\right] d t^{\prime}$

and $\Delta \omega_{j}$ is the detuning of the $j$ th wave from its carrier frequency. Eq. (16) shows that linear propagation over step $\Delta z$ in $\left\{z, \Delta \omega_{j}\right\}$ space is equivalent to shifting the phase of each 
monochromatic wave of frequency shift $\Delta \omega_{j}$ by $\left(\Delta z \Delta \omega_{j}\left[1 / v_{j}-1 / v_{m}\right]\right)$, so linear propagation is handled by transforming to $\{z, \Delta \omega\}$ space: shifting the phase of each monochromatic wave as indicated, and transforming back to $\left\{z, t^{\prime}\right\}$ space. The nonlinear mixing half-step is then performed in $\left\{z, t^{\prime}\right\}$ space by integrating the mixing equations without the group velocity term

$\frac{\partial}{\partial z} \varepsilon_{j}\left(z, t^{\prime}\right)=P_{j}\left(z, t^{\prime}\right)$

over the step $\Delta z$, using a Runge-Kutta method.

Figure 2 illustrates the details of our implementation. The input fields $\varepsilon_{j}^{\text {in }}(t)$ are specified gridded in time. They are modified by the input mirror and input face reflections to give the fields just inside the crystal input face $\varepsilon_{j}\left(t . z_{0}\right)$. As we just described. these fields are Fourier transformed from $\left\{t, z_{0}\right\}$ to $\left\{\Delta \omega, z_{0}\right\}$, the phase shifts are applied. and the fields are transformed back to $\left\{t, z_{0}\right\}$ space. The use of the transformed time $t^{\prime}$ is a. convenience to keep the pulses nearly centered in the time grid. The mixing step then modifies the fields at each point of the time grid according to Eq. (18), giving $\varepsilon_{j}\left(t, z_{1}\right)$. This process is repeated until the final $z$-step in the crystal is reached. The fields exiting the crystal are then delayed on the time grid by the round trip time of the fastest wave and split into components reflected and transmitted by the input mirror. The reflected components are added to the transmitted components of the original input fields and the resulting fields are again passed through the crystal giving results accurate for two crystal passes or over the time interval $\left(0-2 t_{m}^{r t}\right)$. This process is repeated for a third pass giving results accurate over $\left(0-3 t_{m}^{r t}\right)$ and so on until the entire pulse duration is modeled.

We have verified that this method works. However, it is computationally inefficient because the entire time range of the pulses is propagated through the crystal to extend the time history of the pulses by only $t_{m}^{r t}$, requiring many passes with large time arrays. A much more efficient method is to propagate only one round-trip time slice per pass. To find the starting fields for pass $N$, the reflected light from pass $\left(I^{\circ}-1\right)$ is combined with the $N$ th slice of transmitted input light and the entire history is built up one pass at a time. In 
practice, we use time slices somewhat longer than a single round-trip time, specifying the field over the interval $\left([N-1] t_{m}^{r t}-\Delta t\right)$ to $\left(N t_{m}^{r t}+\Delta t\right)$. This is necessary because group velocity differences make the round-trip times different for the different waves. We use the round-trip time of the fastest wave to set the time slices. Propagation of the slower waves causes light from one round-trip slice to flow into the next, so a portion of the preceding slice must be included to accommodate this flow. Additional extra time is used to prevent aliasing in the Fourier transform. This extra interval is usually about 20 time points, and we weight them so the field amplitudes smoothly drop from actual amplitudes at the splice point to zero at the end points. At the end of each round-trip calculation, the spliced-on time zones are dropped, and the remaining field is the calculated field for the $N$ th pass of the cavity. This method assumes that light in the $N^{i}$ th time slice is unaffected by light outside $\forall$. the included time interval, a good approximation so long as there are no sharp features in the spectral response of the crystal that would have a corresponding time transform longer than $\Delta t$. This requirement is almost always met with nonlinear crystals of interest in OPO applications, and is essentially the same assumption made in dropping higher order time derivative terms (group velocity dispersion and higher order dispersion terms) in the mixing equations.

Frequency selective intracavity elements such as etalons can be accommodated in this method even though they might have narrow spectral structure. Reflections within an etalon will introduce time delays that cause some of the light from one OPO round-trip time slice to overflow into later slices. This can be handled by passing the fields covering the full currently known time range from $t=0$ to $t=N t_{m}^{r t}$ through the intracavity element on each pass. Two copies of the fields must be kept. One that has been modified by the element and one that has not. The former is used in the crystal calculations, the latter is passed through the intracavity element to find the new time history after each pass of the cavity.

To illustrate this method, we modeled the OPO of Fig. 1: using the operating parameters listed in Table 1. As a starting point we create an input time grid, identical in size for all three waves, with a time step adjusted to resolve the full acceptance bandwidth of the 
crystal, and a length sufficient to contain the full duration of the input and output pulses. We fill this grid with the applied pump, signal, and idler fields. The signal and idler fields include quantum noise in addition to any seed light. Quantum noise is simulated by filling each longitudinal, or frequency mode of the input grid with light of random phase and with Gaussian amplitude distribution normalized to an average of one photon per longitudinal mode. Enough modes are filled so the spectrum spans the full acceptance bandwidth of the crystal, and they are centered on the carrier frequency of the signal or idler. These fields are Fourier transformed to the time domain and inserted in the input grid. This gives a Gaussian field amplitude distribution at the input mirror with a correlation time less than the inverse crystal acceptance bandwidth. Usually broad-bandwidth OPO's have linewidths three to five times smaller than the crystal acceptance bandwidth, so starting with a noise spectrum slightly broader than the acceptance bandwidth should avoid artificial narrowing of the output spectrum. The time spanned by the input grid is a few times the duration of the pump pulse which in turn is many times the cavity round-trip time, forcing the frequency spacing of the noise modes to be much less than the cavity mode spacing. In this model, the cavity boundary conditions and the crystal gain select the oscillating modes; we make no assumptions about them. We do assume a single Gaussian transverse mode of specified diameter in our simulation. This should be realistic for low-Fresnel-number OPO's which support oscillation on only a single transverse mode.

\section{SPLIT-STEP METHOD 2}

The second model is also based on split-step integration, but rather than transforming between $t$ and $\omega$, we transform between $z$ and $k_{z}$ in the propagation half of the split step. This propagates the fields over equal $t$ steps rather than over equal $z$ steps. In a reference frame with $z$ transformed so the fastest moving pulse is stationary in $z^{\prime}$ : defined by

$z^{\prime}=z-v_{m} t$ 
$t^{\prime}=t$

the propagation equations take the form

$\left[\left(v_{j}-v_{m}\right) \frac{\partial}{\partial z^{\prime}}+\frac{\partial}{\partial t}\right] \varepsilon_{j}\left(z^{\prime}, t\right)=0$

In $\left\{k_{z}, t\right\}$ space they take the form

$\left[i\left(v_{j}-v_{m}\right) \Delta k_{z}+\frac{\partial}{\partial t}\right] \varepsilon_{j}\left(\Delta k_{z}, t\right)=0$

A $z$-grid is set up in the crystal. We define a time step, $\Delta t$, as the time for the fastest wave to move one $z$-step. Grids with this time step are set up as shown in Fig. 3. The input fields are placed on the input grid with time proceeding right to left as shown. Each step in the integration will represent a time snapshot of the fields. Propagation in the crystal proceeds by Fourier transforming all but the fastest wave from $\left\{z^{\prime}, t\right\}$ to $\left\{k_{z}, t\right\}$ over the full crystal length, phase shifting each monochromatic wave by $\left(\Delta k_{z} \Delta t\left[v_{i}-v_{m}\right]\right)$, and transforming back to $\left\{z^{\prime}, t\right\}$. Here, $\Delta k_{z}$ is the offset from the wave vector of the associated carrier wave. This shifts the slower waves to the left, or toward smaller $z^{\prime}$, by the amount $\left(\Delta t\left[v_{m}-v_{i}\right]\right)$. All the fields are then clocked one grid space clockwise over the full cavity and input and output grids, applying the mirror and crystal transmission and reflection coefficients to satisfy boundary conditions. The propagation transform is performed only over the crystal length, rather than over the full cavity. As before, we justify this by noting that ordinarily it is only the light within the crystal that determines the propagation in the crystal. Buffer zones are appended to the crystal fields similar to those described for method 1 above to avoid aliasing in the FFT's.

The mixing half of the split step consists of integrating Eqs. (6-8) from $t_{0}$ to $\left(t_{0}+\Delta t\right)$, nerglecting the propagation term. The mixing equations of form

$\frac{\partial}{\partial t} \varepsilon_{j}\left(z^{\prime}, t\right)=v_{j} P_{j}\left(z^{\prime}, t\right)$

are integrated at each grid point within the crystal. We applied this method under identical conditions to the example above and achieve results identical to those of method 1. 
It is possible to include intracavity etalons in this method directly by adding the etalon mirror reflective boundary conditions to the grid propagation. This is straightforward if the etalon mirrors lie on grid points. If not. FFT methods can be used to shift fields a fraction of a grid spacing to accurately simulate the etalon.

\section{CAVITY-MODE-EXPANSION METHOD}

The cavity-mode expansion method derives exact cavity equations of motion for the intracavity fields by expanding each field in terms of the longitudinal spatial modes of the OPO cavity. This approach has been successfully applied to lasers with externally injected fields ${ }^{25}$. The key step in expanding the fields in a Fourier series of longitudinal modes is to write the d.

field amplitudes inside the cavity as

$\varepsilon_{j}(z, t)=A_{j}(z, t) u_{j}(z) \gamma_{j} \exp \left[-\frac{z}{L} \ln \left(\gamma_{j}\right)\right]+\varepsilon_{j}^{i n j}(t, z)$.

The motivation is to define amplitudes $A_{j}(t, z)$ that are continuous throughout the cavity and satisfy periodic boundary conditions at $z=0$ and $z=L$. The functions $u_{j}(z)$ satisfy $\frac{d u_{j}(z)}{d z}=0$

inside and outside the crystal except at the crystal faces and mirrors where they are discontinuous. They satisfy all boundary conditions at discrete locations in the cavity (mirror reflections, crystal surface losses, and phase shifts upon reflection and transmission at surfaces). Since all the discontinuous boundary conditions inside the cavity are handled by the

functions $u_{j}(z)$, the amplitudes $A_{j}(t, z)$ are continuous between $0<z<L$. At $z=0$ the functions $u_{j}(z)$ satisfy the reduced boundary condition

$$
\sqrt{R_{j}} \exp \left[i \omega_{j} L / c\right] u_{j}(L)=\gamma_{j} u_{j}(0)
$$

The eigenvalues $\gamma_{j}$ are found from the solution of Eq. (26) to be

$$
\gamma_{j}=\sqrt{R_{j}} \sqrt{\left(1-R_{j}^{\text {out }}\right) n_{j}} \sqrt{\frac{1-R_{j}^{\text {in }}}{n_{j}}} \exp \left[i \phi_{j}\right]
$$


with $\phi_{j}$ being the residual round trip phase due to detuning of the carrier from cavity resonance. Thus the functions $u_{j}(z)$ and the constants $\gamma_{j}$ handle discontinuities in the cavity fields for arbitrary mirror reflectivities, crystal reflectivities, and cavity detunings.

In order to make the $A_{j}(t, z)$ 's periodic, it is also necessary to factor out the injected fields as in Eq. (24). These are the fields that develop in the passive cavity due to the input fields so they satisfy the homogeneous plane-wave equation (no nonlinear polarization term) with boundary conditions

$\varepsilon_{j}^{i n j}(t, 0)=\sqrt{1-R_{j}} \varepsilon_{j}^{i n}(t)+\sqrt{R_{j}} \varepsilon_{j}^{i n j}(t, L) \exp \left[i \phi_{j}\right]$

Analytical expressions for $\varepsilon_{j}^{i n j}(t, z)$ are easily obtained for the OPO cavity shown in Fig. 1. Substituting Eq. (24) into the mixing equations, we find the amplitudes $A_{j}(t, z)$ satisfy

$$
\left[\frac{\partial}{\partial z}+\frac{1}{v_{j}}-\frac{1}{L}\right] A_{j}(t, z)=\frac{P_{j}(t, z)}{\gamma_{j} u_{j}(z) \exp \left(-z \ln \gamma_{j} / L\right]}
$$

throughout the cavity, where $v_{j}(z)$ is $v_{j}$ in the crystal and $c$ in air. Using Eqs. (26) and (28), the $A_{j}(t, z)$ 's satisfy the periodic boundary conditions

$A_{j}(t, 0)=A_{j}(t, L)$

at all times. Because they are also continuous functions of $z$; they can be expanded in a Fourier series of longitudinal modes of the empty ring cavity

$$
A_{j}(t, z)=\sum_{q} A_{q}^{j}(t) \exp [-i 2 \pi q z / L]
$$

where $q$ is the cavity mode integer. The expansion coefficients evolve in time according to

$$
\frac{d A_{q^{\prime}}^{j}(t)}{d t}=\frac{1}{L} \sum_{q} V_{q^{\prime}, q}^{j} A_{q}^{j}(t)\left[\ln \gamma_{j}+i 2 \pi q\right]+\frac{1}{L} \int_{0}^{L} d z \frac{\tau_{j}(z) P_{j}(z, t)}{\gamma_{j} u_{j}(z)} \exp \left[\frac{\tilde{z}}{L} \ln \gamma_{j}+i 2 \pi q^{\prime} \frac{z}{L}\right]
$$

where

$$
V_{\eta_{\imath} \eta^{\prime}}^{j}=\frac{1}{L} \int_{0}^{L} d z v_{j}(z) \exp \left[i 2 \pi\left(q^{\prime}-q\right) \frac{z}{L}\right] .
$$

Equation (32) represents the coupled equation of motion for each Fourier component of each field inside the OPO cavity. We solved these equations under identical conditions to 
the example above and achieved results identical to those of methods 1 and 2. This gives us confidence that all three methods solve correctly the three-wave mixing equations with group velocity differences subject to the OPO carity boundary conditions.

Including intracavity etalons is not straightforward in this formulation. In principle, one could find the longitudinal modes of the OPO carity with an etalon in the cavity, and then expand the fields in terms of these modes. However, methods 1 and 2 offer a much more straightforward approach for including etalons. Finally, transverse effects can be included by expanding the fields in the transverse modes of the bare cavity.

\section{COMPARISON OF METHODS}

8.

Each of our models has its own strengths and weaknesses. For a direct comparison among them, we chose the example of Table 1. For method 1, the time step was $0.303 \mathrm{ps}$, the number of time steps included in each single-pass integration was 256. and the number of $z$ steps in the crystal was 50. Run time was about 5 minutes on a $300 \mathrm{~J} / \mathrm{Hz}$ Pentium II computer. For method 2 , the time step was 0.188 ps and the number of $z$ grid points in the crystal was 256. Run time was slightly longer at about 15 minutes. The modeled OPO performance was identical for the two methods. The two run times should each scale approximately the same with changes in bandwidth, pulse duration, crystal and cavity length, so there is not a clear winner in computation speed or memory requirements. Both can be readily extended to include diffraction and birefringent walk off by including in the propagation half-step the transform from $\{x, y\}$ to $\left\{k_{x}, k_{y}\right\}$. Diffraction and walk off are handled by adding the diffractive and walk off phase shifts, followed by transformation back to $\{x, y\}^{19}$. This should apply equally to both spit-step methods. This combination of group-velocity walk off with diffraction and birefringent walk off has been demonstrated by . Wilonni et al. ${ }^{20}$ in a model of single-pass second-harmonic generation that uses the $(t \leftrightarrow \omega)$ transform method to handle group velocity.

If the group velocity dispersion terms in Eqs. (6-8) are important, for example in a 
degenerate type 1 OPO, method 1 has an advantage because the nonlinear mixing equation remains a first order equation, whereas in method 2 it becomes second order. One attractive feature of method 2 is that it gives a series of snapshots of the fields permitting a movie-like display of the fields as they propagate through the crystal. This can provide valuable insight into the evolution of the mixing fields. Implementations of these two methods are included in the public domain software package $\mathrm{SNLO}^{21}$. The cavity mode expansion method is much slower than the other two, with a run time of about 2 hours. The reason for this is not clear, and we did not pursue this method further since methods 1 and 2 give identical results and run much faster. We speculate that one reason method 3 may be slow is because the pump and idler are not resonant in the example calculation yet they are expanded in OPO cavity modes. The expansion coefficients seem to vary rapidly in time requiring small time steps and higher-accuracy integration schemes. We used a fourth-order Runge-Kutta scheme to solve the coupled equations Eq. (42), a time step of $0.076 \mathrm{ps}, 127$ longitudinal modes, and $120 z$-grid points in the crystal for the example of Table 1. Method 3 is of interest primarily as an extension of previous models of multimode lasers and OPO's, showing how they can be adapted to correctly model nanosecond OPO:s.

\section{EXAMPLE RESULTS AND DISCUSSION}

In a pair of earlier papers ${ }^{1,2}$, we presented detailed laboratory measurements of the behavior of two similar KTP ring OPO's. We reported the unseeded linewidths, seed power threshold, and a set of measurements that indicated the unseeded spectrum was nearly phase modulated with little amplitude modulation. It is interesting to compare the predictions of our new models with these observations. The parameters for the KTP OPO are listed in Table 2. First we consider the unseeded spectra. The model predicts large pulse to pulse variation in the signal and idler spectra reflecting the variation in the start up noise. Fig. 4 shows two calculated unseeded spectra under identical operating conditions except for the signal and idler starting noise. This large variation is in qualitative agreement with our laboratory 
observations. Fig. 4 also shows the signal spectrum averaged over 10 pulses. The averaged linewidth is about $2.5 \mathrm{~cm}^{-1}$, in good agreement with the measured linewidth of about 3 $\mathrm{cm}^{-1}$. In these runs the linewidth of the start up noise was $15 \mathrm{~cm}^{-1}$. When $\mathrm{cw}$ seed light at the signal wavelength is added, the spectrum narrows as expected. Fig. 5 shows a series of spectra corresponding to seed levels of $0,1,10$, and $100 \mathrm{nW}$ for the same starting noise in each case. There is slight narrowing with $1 \mathrm{nW}$, but for $10 \mathrm{nW}$ the seeded mode is about 100 time the strength of the other modes, and for $100 \mathrm{nW}$ the seeded mode is about 1000 time the strength of the other modes. The seed light was exactly resonant with a cavity mode. This observation of a seeding threshold power of about $10 \mathrm{nW}$ agrees with our measured seeding threshold ${ }^{1}$ of about $10 \mathrm{nW}$.

The situation with mode correlation or phase modulation is more complex. In our earlier paper we presented a number of measurements that taken together indicated that the light from the KTP OPO was nearly purely phase modulated, although there was no single definitive measurement. We measured directly the signal wave amplitude modulation (AM) at frequencies less than $5 \mathrm{GHz}$, using a fast oscilloscope and detector. This resolved only the $3.66 \mathrm{GHz}$ adjacent mode beating. We found significant AM with pump levels near threshold, but it disappeared as the pump level was increased to 2 and 3 times threshold. We also summed the signal and idler waves in a mixing crystal to create light at the pump wavelength. With no delay between the signal and idler pulses, the reconstructed pump light was nearly monochromatic, but with a delay it developed sidebands. This indicated that the signal and idler waves had large conjugate phase excursions but relatively little AMI. We also frequency doubled the signal wave, comparing doubling efficiency with and without injection seeding. The efficiency changed by $3 \%$ or less indicating that the output signal light had much less AM than the quantum noise from which oscillation builds. From this experimental evidence we conclude that, unlike many Q-switched lasers, an OPO does not amplify the starting noise with weak correlations between modes to give nearly chaotic light. but rather produces light in several longitudinal modes with strong intermode correlations. The light behaves more like phase modulated (P.I) than chaotic light. In our earlier paper. 
we also presented a model calculation that pointed toward pure PM light. The model started with two signal cavity modes seeded so the seed had strong AM. We integrated the mixing equations without the group velocity terms and found that the AM was strongly suppressed at high pump levels but not near threshold, in qualitative agreement with experiment. We have now applied our new models that include the group velocity terms to address the question of the correlation between modes. Based on a limited number of model runs, we find strong suppression of amplitude modulation in a signal-resonant OPO if the group velocity of the pump is midway between those of the signal and idler. With increasing deviation from that value, the amplitude modulation tends to grow. Interestingly, this optimum value corresponds to exact phase matching for frequency summing pairs of signal and idler sidebands with equal detuning from their carriers. That is, all processes satisfying $\left(\left[\Delta \dot{\omega}_{s}=N \delta\right]+\left[\Delta \omega_{i}=N \delta\right] \rightarrow\left[\Delta \omega_{p}=+2 N \delta\right]\right)$ are exactly phase matched. Why this should suppress AM most effectively is not obvious. This case is illustrated in Fig. 6 which shows time profiles of the signal wave for various pump levels for the OPO of Table 1. Each run uses the same starting noise. The suppression of AM with increasing pump is apparent. Further, the leading edge of the signal pulses retains some modulation even at the higher pump levels, qualitatively agreeing with our laboratory observations. The details of the leading-edge modulation reflect the modulation in the start-up noise fields and fluctuate from pulse to pulse if different starting noise is used.

However in the KTP OPO's of our earlier papers, the pump group velocity is slower than both the signal and idler velocities. Our model indicates that AM suppression is weaker here. In fact, rather than producing a smooth time profile the model predicts a smooth profile interrupted by amplitude excursions with the characteristic shape shown in Fig. 7. The location and abundance of these blips depends on the starting noise but their shape is nearly universal. They persist for the duration of the pulse, recurring with a round trip period. They are a direct consequence of the difference in group velocities of the three waves and their width approximates the pump/idler walk off time $\tau_{i p}$ of $4.9 \mathrm{ps}$. Different sets of group velocities develop other characteristic shapes. Interestingly, when we calculate 
the relative efficiency for frequency doubling the predicted signal wave, it is within $3 \%$ of a temporally smooth pulse, so the second harmonic test of our previous work which had a $3 \%$ detection limit would not have clearly detected this type of AM. Further, when we filter the signal wave as detected by a square law detector to pass only frequencies less than 5 $\mathrm{GHz}$, corresponding to our direct AM measurement, very little modulation appears on the pulse profile because the blips are bipolar with nearly equal positive and negative excursions. Also, near threshold the characteristic shape is replaced by only negative excursions so more AM is predicted. This trend is in qualitative agreement with our earlier report. However, as the pump level is increased to 4 times threshold the number of blips increases as does the $3.66 \mathrm{GHz} \mathrm{AM}$, in disagreement with laboratory observations. We must conclude that while there is strong correlation between modes, perhaps AM suppression is not complete in all cases, including the KTP OPO's we studied. We note, however, that a few percent idler feedback significantly reduced the number of blips. Also, the velocity of the blips slows slightly with increasing pump level so it may be that including realistic transverse beam profiles and diffraction would reduce or eliminate them.

Another testable feature predicted by our model is the existence of many low level sidebands on the depleted pump light. A predicted power spectrum shown in Fig. 8 for the KTP ring OPO has many sidebands at the sub-percent level spread over a range approximately equal to the $8 \mathrm{~cm}^{-1}$ crystal acceptance bandwidth, with peaks separated by the OPO mode spacing, $\delta$. For seeded operation, these sidebands are absent. We have verified this behavior in a laboratory OPO by analyzing the depleted pump light using a Fabry-Perot etalon. Fig. 9 compares depleted pump spectra for seeded and unseeded operation along with a reference spectrum taken by blocking the signal wave inside the OPO cavity to prevent oscillation. Note the presence of many low level modes only in the unseeded case. Our analyzing FabryPerot had a free spectral range of $45.4 \mathrm{GHz}$ and finesse of about 35. We scanned the central spot of the etalon with each point on the trace representing the average over approximately 10 pulses. The sidebands on the depleted pump extend beyond the free spectral range of the etalon so each side band is actually a sum of sidebands associated with different etalon 
orders. The etalon spacing was adjusted to overlap the orders. From a separate etalon scan with the free spectral range extended to $6 \mathrm{~cm}^{-1}$. we find the sidebands extend at least 5 $\mathrm{cm}^{-1}$ on each side of the input pump frequency. The individual sideband energies are in the range of $0.3 \%$ to $1 \%$.

\section{CONCLUSIONS}

We demonstrated three methods of modeling broad-bandwidth nanosecond OPO's in the plane-wave limit and show excellent agreement among them. We pointed out the advantages and limitations of each and indicated how they could be modified to include diffraction and birefringent walk off as well as how intracavity frequency selective elements could be included. The models predict unseeded. OPO's generate light with unusual spectral properties. It is broad band but has much less amplitude modulation than it would if random phases were assumed for the longitudinal modes. Evidently the modal phases are locked in such a way that they produce nearly phase modulated light, in accord with our earlier laboratory observations ${ }^{2}$. We also find that the group velocity of the pump light relative to those of the signal and idler has an impact on the spectral properties of an OPO. With a pump velocity intermediate between signal and idler velocities. Aiv suppression is strongest. Additionally, the output waves tend to develop characteristic blips whose shape depend on the relative group velocities. We expect these models will proride a guide in designing nanosecond OPO's with spectral control either by injection seeding or by use of intracavity frequency selective elements. Although we have not presenred examples here, our models also permit the use of multimode pump light, so the influence of the pump spectrum can also be studied. Finally, we note that method 1 can readily accommodate group velocity dispersion terms so it should be possible to model degenerate type 1 OPO's with their extremely wide bandwidth. 


\section{REFERENCES}

1. A. V. Smith, W. J. Alford, T. D. Raymond, and M. S. Bowers, "Comparison of a numerical model with measured performance of a seeded, nanosecond KTP optical parametric oscillator," J. Opt. Soc. Am. B 12, 2253-2267 (1995).

2. D. J. Armstrong and A. V. Smith, "Tendency of nanosecond optical parametric oscillators to produce purely phase-modulated light," Opt. Lett. 21, 1634-1636 (1996).

3. Y. R. Shen, The Principles of Nonlinear Optics (Wiley \& Sons New York, 1984).

4. E.S. Cassedy and M. Jain, "A theoretical study of injection tuning of optical parametric oscillators," IEEE J. Quant. Electron. QE-15, 1290-1301 (1979). i.

5. Y. Kong, Z. Xu, Y. Zhou, D. Deng, X. Zhu, and L. Wu, "The compound cavity optical parametric oscillator: theory and experiment," IEEE J. Quant. Electron. 34, 439-446 (1998).

6. T. Schroder, K.-J. Boller, A. Fix, and R. Wallenstein, "Spectral properties and numerical modeling of a critically phase-matched nanosecond $\mathrm{LiB}_{3} \mathrm{O}_{5}$ optical parametric oscillator," Appl. Phys. B 58, 425-438 (1994).

7. K.-J. Boller and T. Schroder, "Demonstration of broadband intracavity spectroscopy" in a pulsed optical parametric oscillator made of $\beta$-barium borate," J. Opt. Soc. Am. B 10, 1778-1784 (1993).

8. A. Fix and R. Wallenstein, "Spectral properties of pulsed nanosecond optical parametric oscillators: experimental investigation and numerical analysis," J. Opt. Soc. Am. B 13, 2484-2497 (1996).

9. A. Yariv and W. H. Louisell, "Theory of the optical parametric oscillator," IEEE J. Quant. Electron. QE-2, 418-424 (1966).

10. T. Debuisschert, "Nanosecond optical parametric oscillators," Quant. Semiclass. Opt. 
9, 209-219 (1997).

11. K. D. Shaw, "Spatio-temporal evolution of the intra-cavity fields in a pulsed doubly resonant optical parametric oscillator," Opt. Comm. 144, 134-160 (1997).

12. G. Arisholm, "Advanced numerical simulation models for second-order nonlinear interactions," to appear in SPIE proceedings of Laser Optics '98 Conf., St. Petersburg, June 1998.

13. H. J. Bakker, P. C. M. Planken, and H. G. Muller, "Numerical calculation of optical frequency-conversion processes: a new approach," J. Opt. Soc. Am. B 6, 1665-1672 (1989).

14. M. Cavallari, G. M. Gale, F. Hache, L. I. Pavlov, and E. Rousseau, "Mid infra-red femtosecond pulse generation by wave-mixing: numerical simulation an experiment;," Opt. Comm. 114, 329-332 (1995).

15. R. Danielius, A. Dubietis, A. Piskarskas, G. Valiulis, and A. Varanavicius, "Generation of compressed $600-720-\mathrm{nm}$ tunable femtosecond pulses by transient frequency mixing in a $\beta$-barium borate crystal," Opt. Lett. 21, 216-218 (1996).

16. R. Danelyus, G. Dikchyus, V. Kabelka, A. Piskarskas, A. Stabinis, and Ya. Yasevichyute. "Parametric excitation of light in the picosecond range:" Sov. J. Quant. Electron. 7, 1360-1368 (1977).

17. S. Fournier, R. Lopez-Martens, C. Le Blanc, E. Baubeau, and F. Salin, "Solitonlike pulse shortening in a femtosecond parametric amplifier." Opt. Lett. 23, 627-629 (1998).

18. D. Kim and G.-Y. Xiao, "Distortion of a chirped short pulse in type II second-harmonic generation," J. Opt. Soc. Am. B 15, 570-576 (1998).

19. A. V. Smith and M. S. Bowers, "Phase distortions in sum- and difference-frequency mixing in crystals," J. Opt. Soc. Am. B 12, 49-57 (1995). 
20. P. W. Milonni, J. M. Auerbach, and D. Eimerl, "Frequency conversion modeling with spatially and temporally varying beams," SPIE 2633, 230-241 (1997).

21. SNLO nonlinear optics software is available from A. V. Smith, Dept. 1128 Sandia National Laboratories, $87185-1423$ or it may be downloaded from www site http://www.sandia.gov/imrl/XWEB1128/xxtal.htm. Methods 1 and 2 are run by the Run and Movie buttons of function PW-OPO-BB respectively.

22. T. Nishikawa and N. Uesugi, "Effects of walk-off and group velocity difference on the optical parametric generation in $\mathrm{KTiOPO}_{4}$ crystals;" J. Appl. Phys. 77, 4941-4947 (1995).

23. T. Nishikawa and N. Ueşugi, "Transverse beam profiles on traveling-wave optical parametric generation in $\mathrm{KTiOPO}_{4}$ crystals," J. Appl. Phys. 78, 6361-6366 (1995).

24. G. M. Gale, M. Cavallari, and F. Hache, "Femtosecond visible optical parametric oscillator," J. Opt. Soc. Am. B 15, 702-714 (1998).

25. M. S. Bowers and S. E. Moody, "Cavity equations for a laser with an externally injected signal," J. Opt. Soc. Am. B 11, 2266-2275 (1994). 


\section{FIGURES}

Fig. 1. Diagram of the optical parametric oscillators modeled in this paper. The reflectivities of mirrors M2 and M3 are 1.0 for all three waves. Mirror M1 reflectivities and other parameters are given in Tables 1 and 2.

Fig. 2. An example of $\mathrm{OPO}$ model gridding for split-step method 1. The pulses are gridded in time at each $z$ point.

Fig. 3. An example of OPO model gridding for split-step method 2. The $z$ grids outside the crystal are spaced by $\Delta z=c / \Delta t$, that in the crystal is spaced by $\Delta z=v_{\max } / \Delta t$ where $v_{\max }$ is the fastest of the three group velocities.

Fig. 4. Signal spectra for $O P . O$ of Table 2 pumped at twice threshold. Top two traces are for individual pulses with different start up noise. Bottom trace is the average of ten pulses with fluctuating start up noise. The averaged spectral width is about $2.5 \mathrm{~cm}^{-1}$ compared with the crystal acceptance bandwidth of $8 \mathrm{~cm}^{-1}$.

Fig. 5. Signal spectra for OPO of Table 2 pumped at twice threshold. The cw seed power incident on the input mirror is varied from 0 to $100 \mathrm{nW}$ with the same start up noise for each pulse. The spectra are normalized so the sum of the energy in all modes is unity: Note the scale changes between the graphs.

Fig. 6. Time profiles of the signal wave for the OPO of Table 1 with pump pulse fluences near threshold, twice threshold and four times threshold. The pump pulse is centered a time zero and has a 7 ns width (FWHM).

Fig. 7. Time profile of the signal wave of the OPO of Table 2 pumped at twice threshold. One half of a cavity round trip is displayed starting about midway through the signal pulse.

Fig. 8. Computed spectrum of the depleted pump wave for the OPO of Table 2 pumped at twice threshold. 
Fig. 9. Measured spectra of the depleted pump wave for unseeded (top trace) and seeded (middle trace) OPO of Table 2. The bottom trace is a reference trace with the signal path blocked inside the OPO cavity. Each sideband is the overlap of sidebands from several etalon orders. The etalon free spectral range is $45.4 \mathrm{GHz}$ and the OPO cavity free spectral range is $4.1 \mathrm{GHz}$. The peaks at +2 and $+14 \mathrm{GHz}$ are measurement artifacts.

४. 


\section{TABLES}

Table 1. Parameters of Example OPO

\begin{tabular}{lccc}
\hline Parameter & Pump & Signal & Idler \\
\hline Wavelength (nm) & 355 & 710 & 710 \\
Refractive Index & 2.2 & 2.2 & 2.2 \\
Group Velocity Index & 2.275 & 2.2 & 2.35 \\
Crystal Absorption (cm $\left.{ }^{-1}\right)$ & 0 & 0 & 0 \\
Input Energy (J) & 0.4 & quant noise + variable seed & quant noise \\
FWIHM Pulse Duration (ns) & 2 & 4 & 4 \\
FWHM Beam Diameter (mm) & 4. & 0.7 & 0 \\
Input Mirror Reflectivity & 0 & Crystal Length (mm) & 5 \\
\hline Cavity Length (mm) & 11 & $\Delta k$ (mm $\left.{ }^{-1}\right)$ & 0 \\
deff (pm/V) & 2 & & 5 \\
\hline
\end{tabular}


Table 2. Parameters of KTP Ring OPO

\begin{tabular}{|c|c|c|c|}
\hline Parameter & Pump & Signal & Idler \\
\hline Wavelength (nm) & 532 & 800 & 1588 \\
\hline Refractive Index & 1.79 & 1.818 & 1.736 \\
\hline Group Velocity Index & 1.911 & 1.875 & 1.765 \\
\hline Crystal Absorption $\left(\mathrm{cm}^{-1}\right)$ & 0 & 0 & 0 \\
\hline Input Energy $(J)$ & 0.4 & quant noise + variable seed & quant noise \\
\hline FWHM Pulse Duration (ns) & 7 & & \\
\hline FWHM Beam Diameter (mm) & 0.6 & 0.6 & 0.6 \\
\hline Input Mirror Reflectivity & 0 & 0.84 & 0 \\
\hline Cavity Length (mm) & 73 & Crystal Length (mm) & 10 \\
\hline $\mathrm{d}_{\text {eff }}(\mathrm{pm} / \mathrm{V})$ & 3.1 & $\Delta k\left(\mathrm{~mm}^{-1}\right)$ & 0 \\
\hline
\end{tabular}




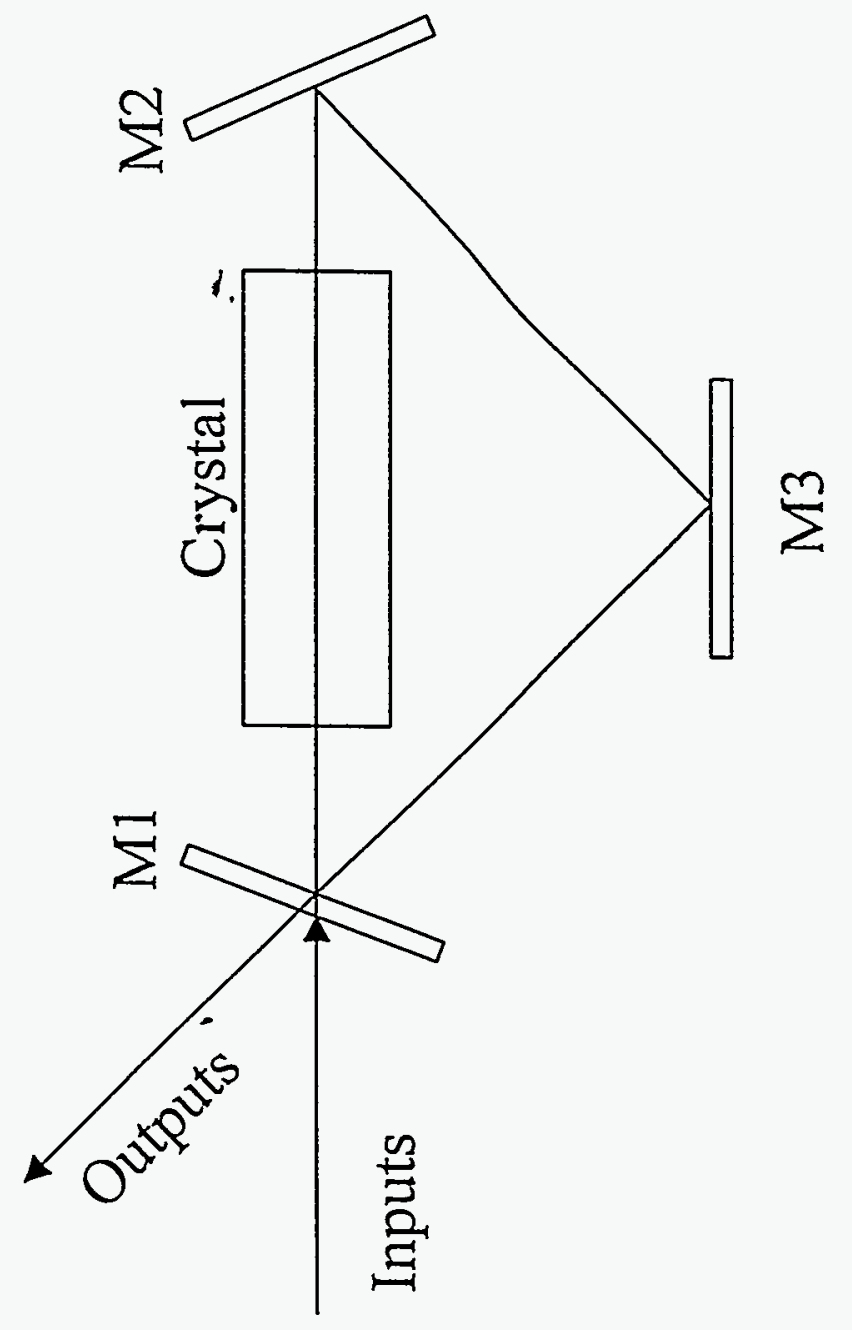




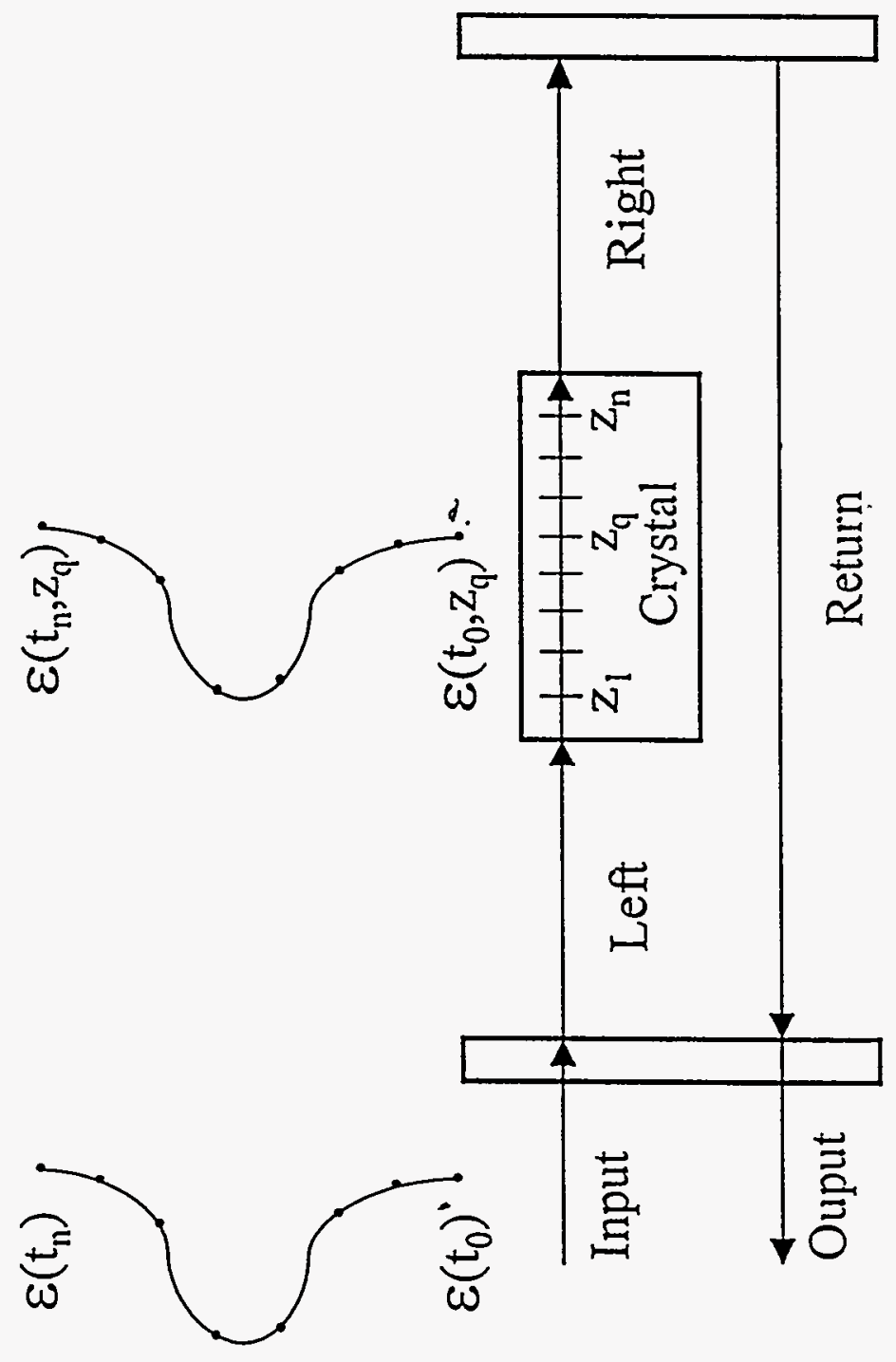




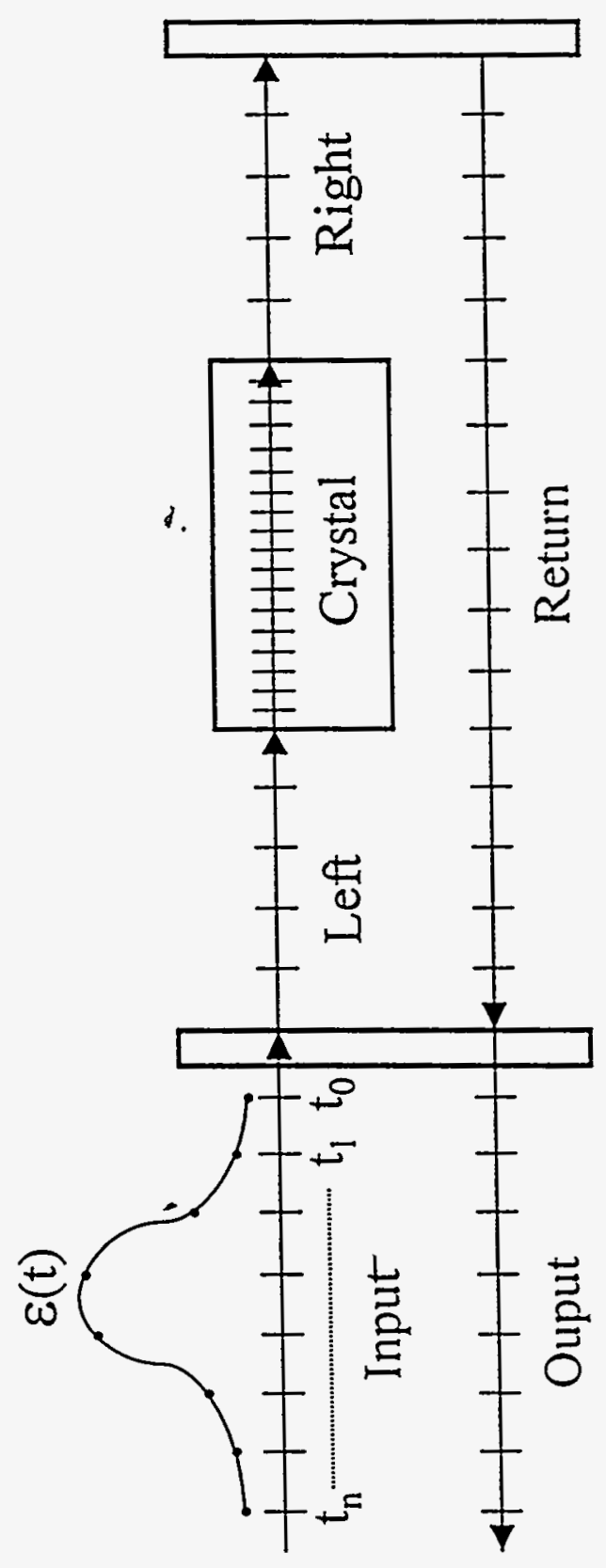




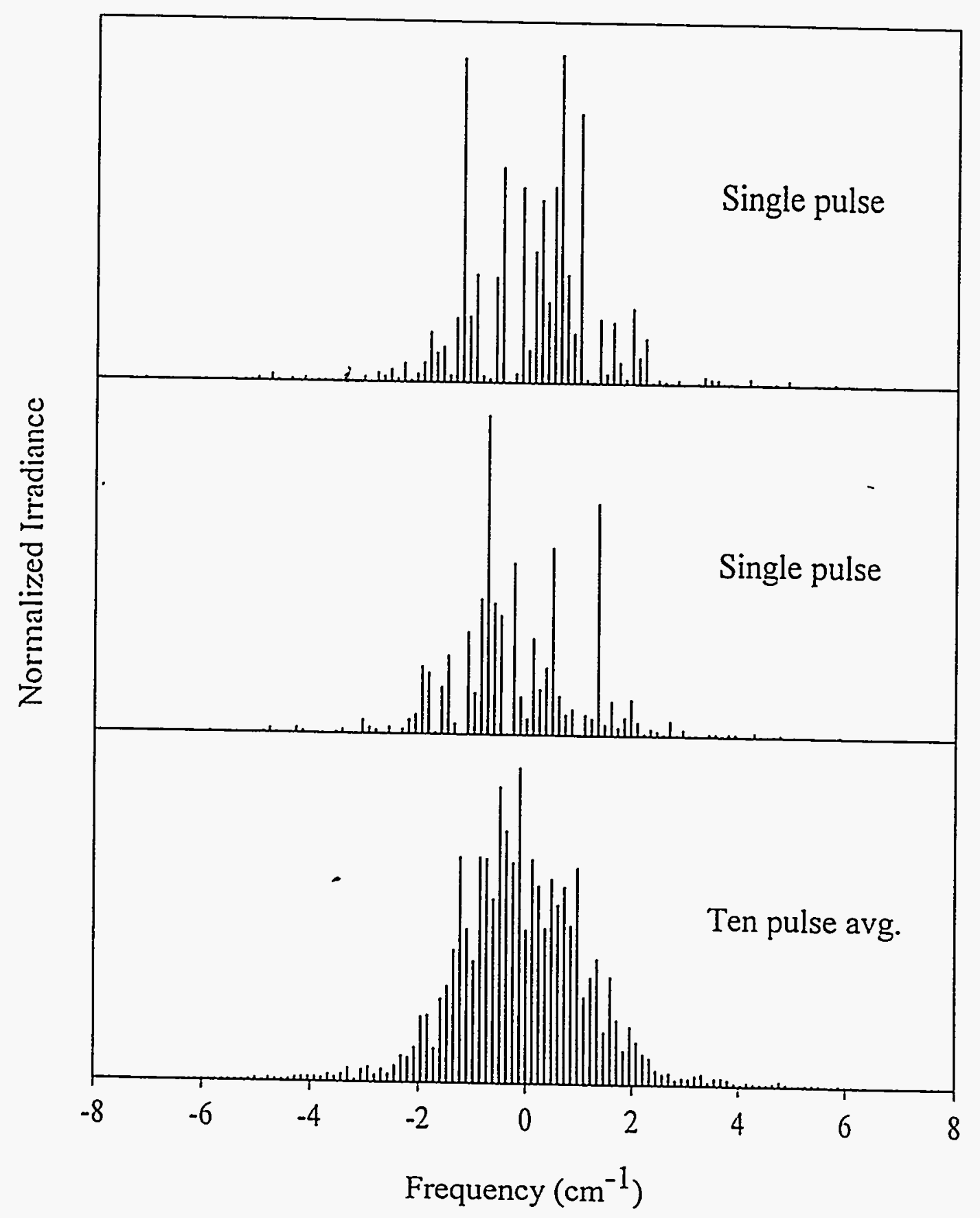



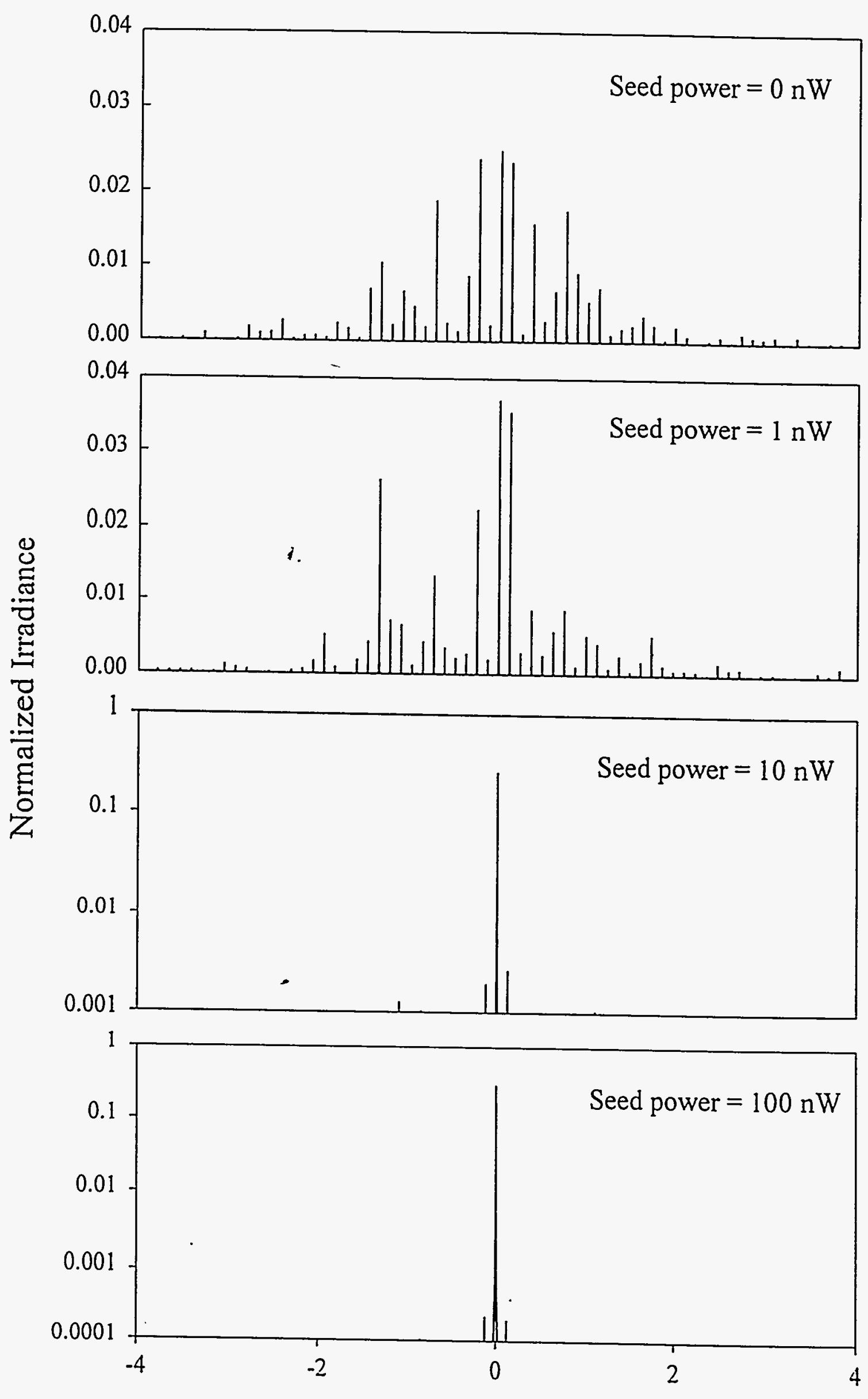

Freauency $\left(\mathrm{cm}^{-1}\right)$ 


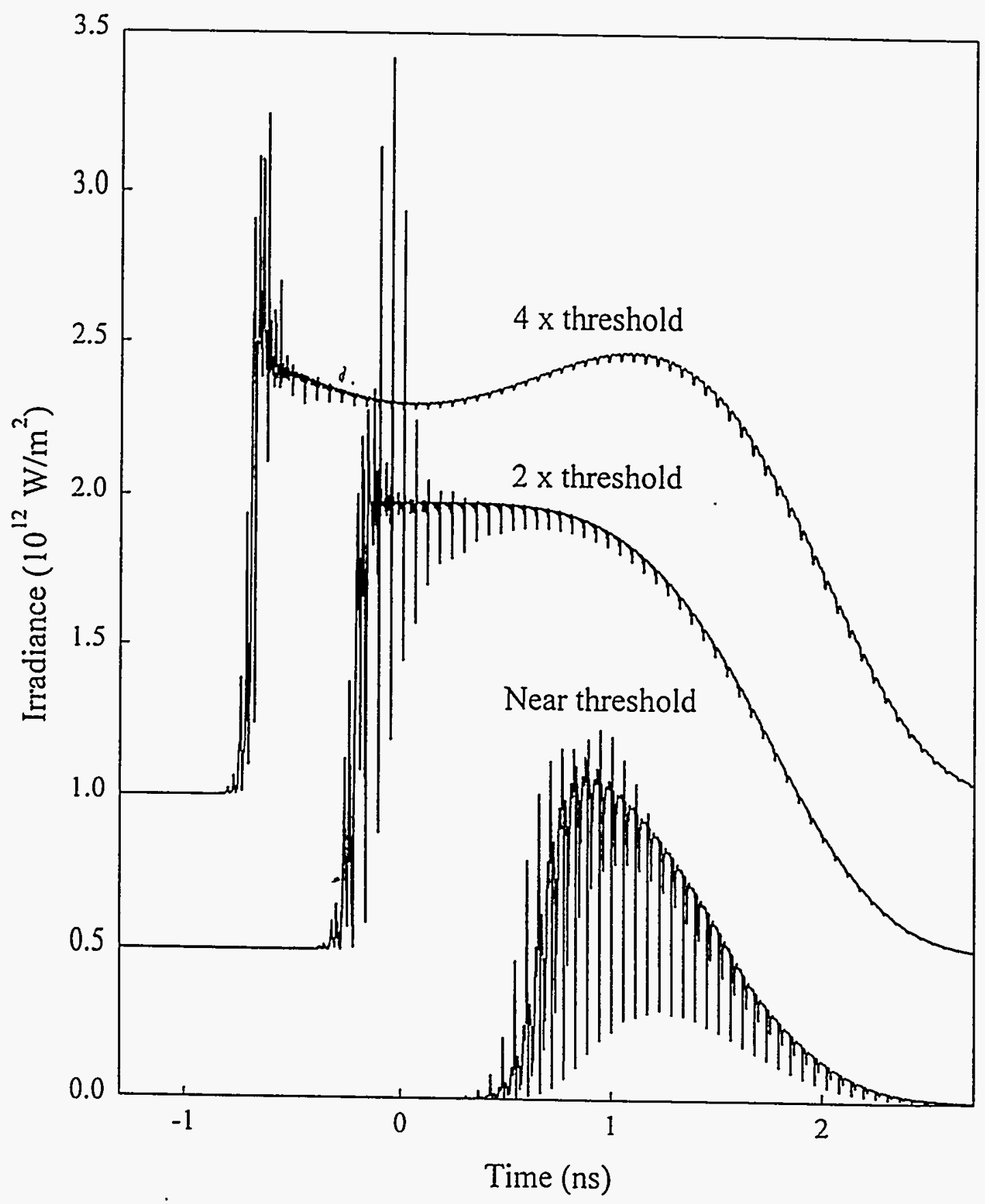




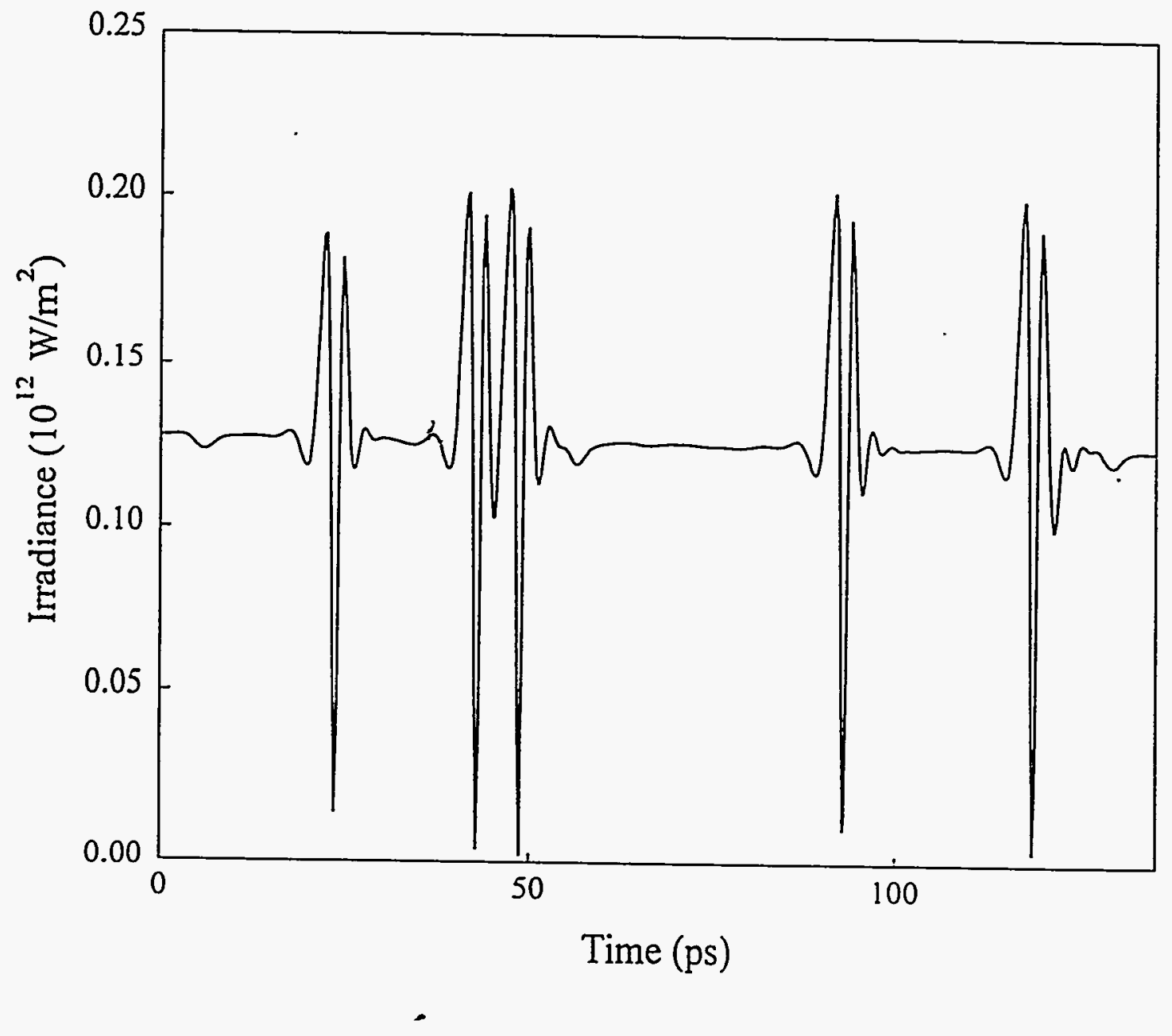




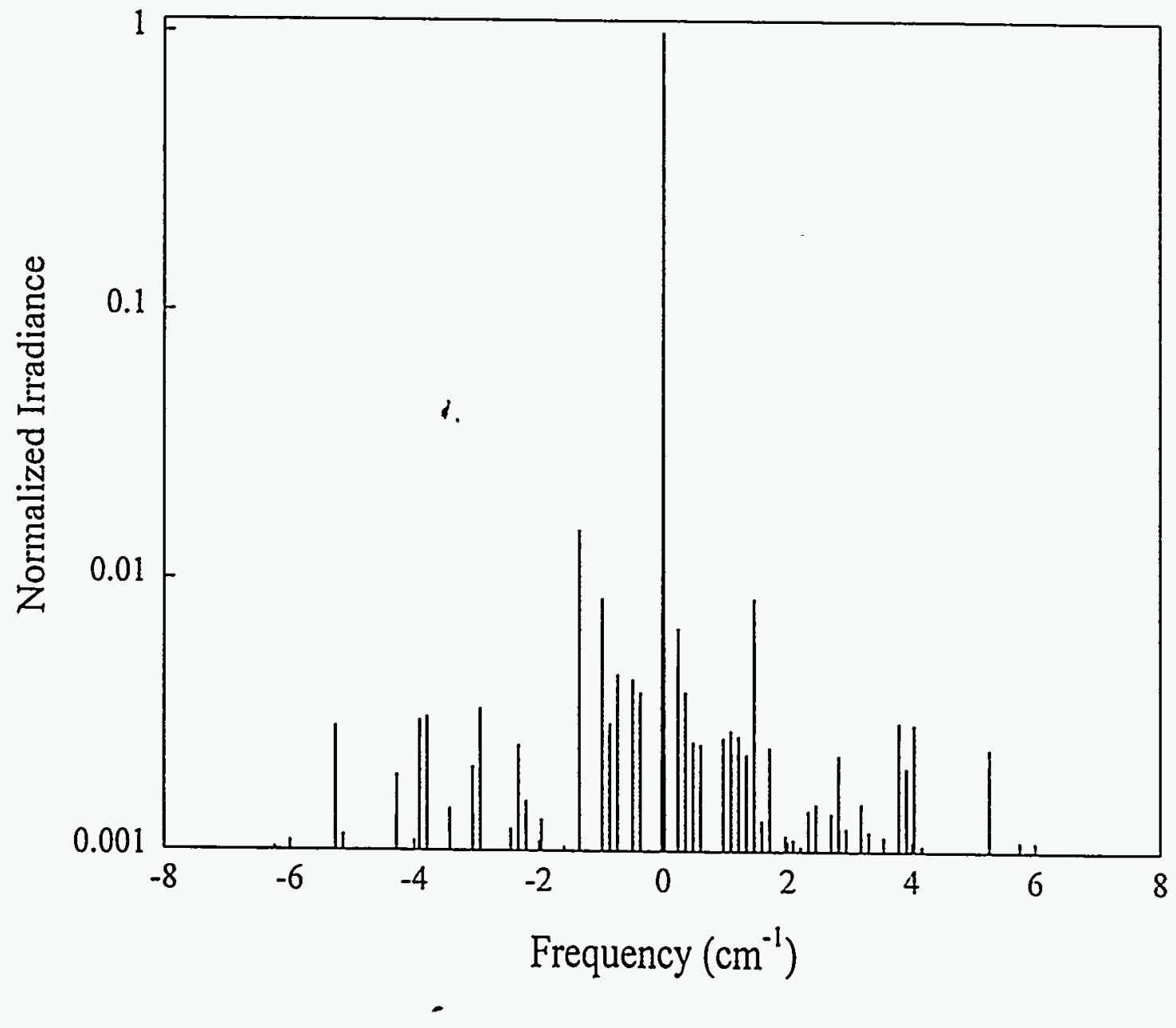




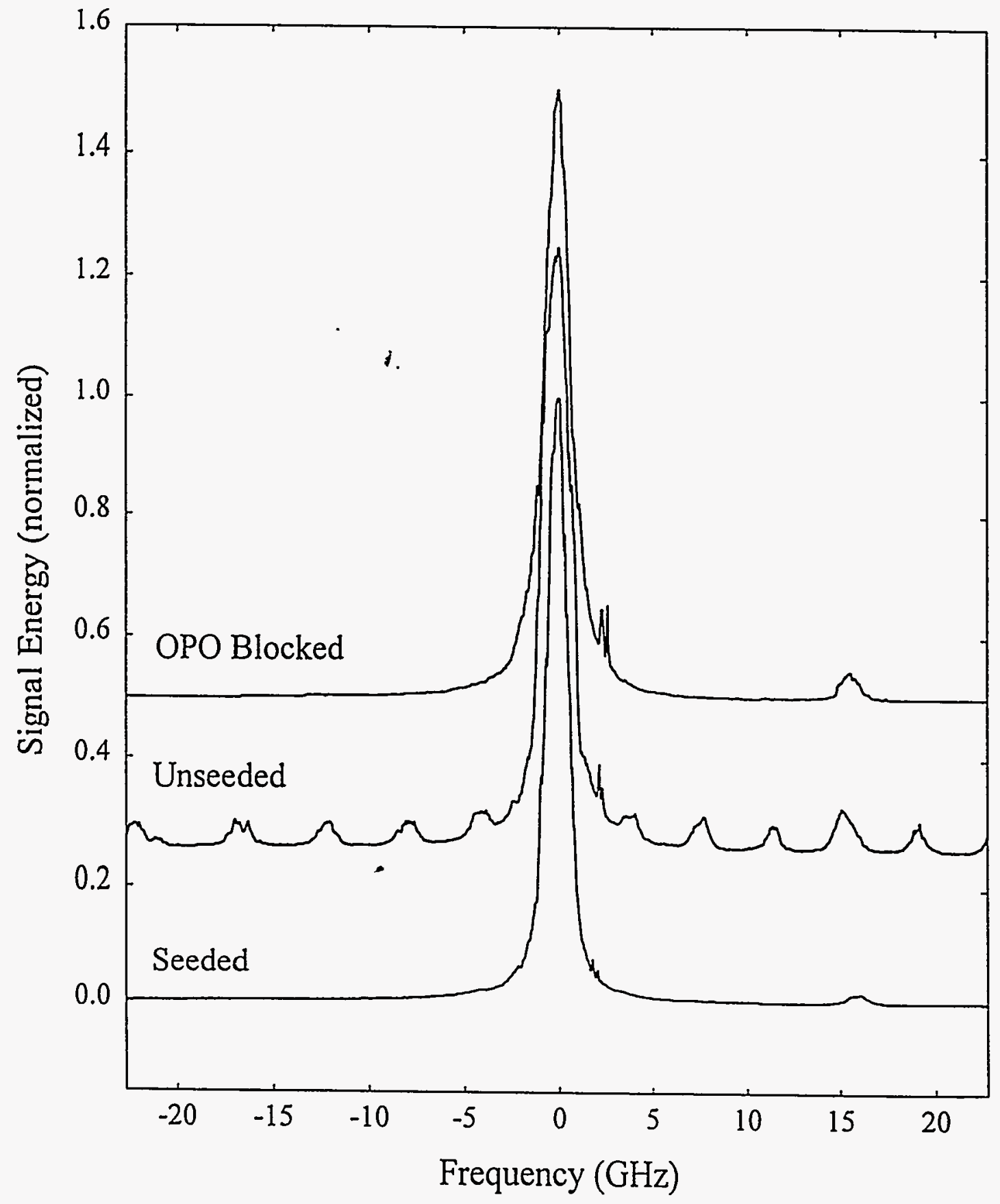

